\title{
Entre puentes y muros. El consejo de defensa sudamericano y las identidades estratégicas de Brasil y Argentina
}

\section{Between bridges and walls. The South American defense council and the strategics identities of Brazil and Argentina}

Rev. Bra. Est. Def. v. 4, nº 1, jan./jun. 2017, p. 61-82

DOI: $10.26792 /$ RBED.v4n 1.2017 .65320

ISSN 2358-3932

\section{CRISTIANO ARMANDO DINIZ GUERRA SILVESTRE ÉRICA CRISTINA ALEXANDRE WINAND}

\section{CONSIDERACIONES INTRODUCTORIAS}

De acuerdo con Saint-Pierre y Lopes (Carmo et al. 2013, 285), "un concepto categoriza, define, limita, la realidad en torno a una hipótesis formulada por un ser histórico y social”. ${ }^{1}$ Así, las relaciones que los países de la región pueden ejercer pasan esencialmente por la definición de conceptos y concepciones, o, en otras palabras, la manera como un país percibe las cosas.

Soares (2013) señala que "las políticas de defensa de Argentina y Brasil revelan distinciones entre el estatuto de la cooperación" relevantes para la comprensión de los descompases entre los dos. El autor considera que las políticas de defensa de ambos los países muestran trazos de una identidad recíproca, es decir, constituida mutualmente por la percepción del "yo" y del "otro", y que una de las fuentes para reconocimiento de esto es el discurso que, según él, es un "revelador de percepciones". No es nuestra intención trabajar con métodos de análisis de discurso, pero examinar distintos posicionamientos de Defensa presentes en los documentos enunciativos de los dos países, pasando por la decurrentes distintas atribuciones de misiones a las Fuerzas Armadas en los dos Estados, a fin explicar porque aquellos enunciados pueden dificultar el desarrollo de una "cultura estratégica conjunta”. ${ }^{2}$

Parte significativa de lo que conduce uno Estado a desarrollar voluntad cooperativa de largo plazo es explicada por la existencia de amenazas

Cristiano Armando Diniz Guerra Silvestre - COPEDE.

Érica Cristina Alexandre Winand - COPEDE, Universidade Federal de Sergipe. 
mutuamente percibidas. Sin embargo, estamos tratando de un universo de construcciones intersubjetivas, ya que como preconiza Héctor Saint-Pierre (2011), la amenaza, punto referente de la defensa, no tiene su existencia en sí misma, pero si en la percepción de quien se siente amenazado. Esto implica en decir que la amenaza tampoco es un objeto en sí mismo, pero deriva de la dinámica perceptiva relacional establecida entre el emisor de la señal y su receptor. El autor aún argumenta que la seguridad es de la misma materia empírica que las percepciones: que es sobre la percepción de los otros que actúa la política externa y, por consiguiente, la defensa, cómo veremos. Siendo así, no debemos esperar que convergencias conceptuales ocurran espontáneamente. ${ }^{3}$ Conforme observan Saint-Pierre y Lopes (2013, 285):

[… ni todos los actores regionales comprenden el mismo por "defensa" "seguridad" o mismo "cooperación". Las diferencias históricas, geopolíticas, culturales, idiosincráticas, institucionales, de percepciones de amenazas y sus capacidades defensivas hacen parte de la explicación de esta polifonía. No obstante, los entendimientos que se suponen la base de cualquier negociación, proceso de negociación o proceso de cooperación exigen como condición de posibilidad [...] de univocidad conceptual, es decir, que todos atribuyan a cada concepto, y solamente a él, una y solamente una semántica.

Poniendo el argumento en esta orden, es posible entender que el intento de traer el "nosotros" al centro de la discusión, en lugar de "yo" y el "otro", se vuelve inviable cuando de frente a la "Torre de Babel" conceptual, como puesto arriba por Saint-Pierre y Lopes. No obstante, en el ámbito del Consejo de Defensa Sudamericano (CDS) de la Unión de las Naciones de la América del Sur (Unasur) se ha intentado, conciliar, o por lo menos aproximar, los entendimientos de los países en una conceptualización común, especialmente con la creación del Centro de Estudios Estratégicos (CEED) del CDS/Unasur que abraza como una de sus misiones primordiales la discusión semántica de los conceptos centrales en el entendimiento regional de la Defensa y Seguridad. Se trata, por lo tanto, de un intento de construcción de identidad estratégica compartida, a partir del diálogo y del compromiso regional en abalizar la mencionada identidad estratégica regional. Defendemos que a partir del alcance de puntos mínimamente comunes, se pueda reverter las miradas normativas y que estas, por su vez, sean capaces de dirimir las cuestiones muy dispares entre los distintos países, de modo que los actores que ejecutan estos nuevos postulados puedan vivir la experiencia y dar funcionalidad a los ajustes conceptuales, y que con en el pasar del tiempo se apropien de esta nueva cultura. 
La observación de las identidades estratégicas pasa, según Luis Tibiletti (2014), por la atribución de un patrón de respuestas a las siguientes preguntas: 1 ¿Cómo el Estado interpreta su entorno estratégico y la relación conflicto-cooperación intrínseca a él? 2. ¿Cómo el Estado se ve a sí mismo dentro de aquellos términos? 3. ¿Cuáles son los intereses propios y cuáles de ellos se está dispuesto a compartir? ¿Por cuales intereses se dispondría a usar la violencia armada? 4. ¿Contra quién y contra qué el Estado piensa en se defender? 5. ¿Con quién se imagina cooperando en términos de defensa? 6. ¿Cómo piensa en se defender? (Tibiletti 2014).

Sin una mínima coincidencia en las respuestas estatales que pueden ser empíricamente atribuidas a las preguntas arriba, queda limitado el horizonte de la identidad estratégica en cuanto pilar de la arquitectura cooperativa regional. No obstante, aun recorriendo a Tibiletti (2014), es importante discernir en que son muchas las perspectivas conceptuales de identidad. Para el autor, solamente tiene sentido investigar hasta qué punto las modificaciones en las concepciones prácticas pueden modificar identidades estratégicas, cuando las mismas son concebidas como en permanente construcción y nunca totalmente definidas, como también sujetas a nuevos significados frente a nuevos contextos y demandas, en constante y dinámica interrelación entre discursos identitários y auto reconocimiento efectivo de los actores en sus prácticas diarias. El autor evoca, de esta manera, por un lado la concepción de Wendt (1994) según la cual "cambiando las prácticas cambiará el conocimiento intersubjetivo que constituye las identidades" como también el concepto de Habermas (apud Tibiletti 2014) de la identidad, no como algo dado, pero "también y simultáneamente nuestro propio proyecto".

Por otro lado, identidades estratégicas son condicionadas por culturas estratégicas que involucran diferentes grupos de actores nacionales y la relación de aquellos actores internacionales. Uno de los grupos principales que contiene su propia carga valorativa son los militares. En este sentido, Adriana Marques (2007) defiende que es necesario que elementos culturales sean apropiados e interpretados por los militares. A ejemplo de eso, la autora menciona el pensamiento de los militares brasileños sobre la Amazonia como condicionado por un "repertorio" de experiencia históricas de las fuerzas armadas en la región.”, y concluye que "en suma, la cultura estratégica de un grupo social es moldada por la práctica de sus miembros bien como la práctica de los miembros de un grupo social es moldada por la cultura estratégica de este grupo" (Marques 2007, 45). Contemplados por el raciocinio de Marques, entendemos que las distintas concepciones reflejadas en el marco legal, así como las distintas atribuciones de misiones a las fuerzas armadas de Brasil y Argentina caracterizan obstáculos al pro- 
yecto unasurino de una "identidad regional compartida”. En otra palabras, la atribución de misiones a las Fuerzas Armadas constituye variable-clave si tenemos en cuenta que a partir de ellas aquél grupo de actores centrales a la defensa molda su visión y sus valores, a través de experiencias vividas.

Dicho esto, en el próximo apartado, de manera ilustrativa buscaremos componer el mosaico regional y la propuesta regional de defensa en lo que nombramos "Entre lo que propone el CDS y lo que existe en la región". En seguida, profundizaremos el debate a través de la discusión teórica fundante de la discusión: la fuerza, y la variedad de formas aplicarla según las distintas percepciones estatales, mirando con un poco más de profundidad los dos casos que trabajemos en esta oportunidad en la sección "Del monopolio de la fuerza a las distintas comprensiones estatales de su uso", enfocaremos el debate en los casos de Brasil y Argentina”. Tenemos presente que no lograremos alcanzar todos los temas de la discusión y tampoco con todo los detalles posibles que nos gustaría, pero en la última parte de esto trabajo, teniendo los pantallazos macro y micro de la parte del proyecto unasurino que le toca al CDS, presentar nuestros entendimientos acerca de los obstáculos que existen para el cumplimiento de sus objetivos.

\section{ENTRE LO QUE PROPONE EL CDS Y LO QUE EXISTE EN LA REGIÓN}

La América del Sur se tiene comprehendida como el espacio en que las relaciones políticas, económicas y estratégicas presentan cierta cercanía en temas y agendas, y este esfuerzo de congregación dio origen a la Unión de las Naciones Sudamericanas (Unasur). En las palabras de Pablo Celi, "la América Latina y, particularmente Suramérica, entró en un proceso de reestructuración de sus relaciones regionales, articulando nuevos direccionamientos políticos y económicos con una reorientación de proyección estratégica en materia de seguridad regional y defensa”.

Para aquel autor, las dinámicas políticas que dan impulso a este proceso están centradas principalmente en la recuperación del papel de los estados y en la inter-relación que estos pueden asumir como herramienta del posicionamiento de sus políticas externas. Celi sigue argumentando que "a estas iniciativas políticas y económicas se asocia un nuevo tipo de proyección estratégica, principalmente en Suramérica, que busca posicionar la región en el mundo, ampliando su contex to multilateral y elevando el papel de los estados de la región en el sistema internacional”.

Oficializada en 2008, cuando de la firma del Tratado Constitutivo de la Unasur, según su artículo $3^{\circ}$, es una organización conformada por doce países de la región - siendo el único país ausente la Guyana Francesa. La organización se autodefine como un organismo internacional cuyo obje- 
tivo es construir un espacio de integración cultural, económica, social y política que respeta la realidad de cada nación. Y que su desafío es "eliminar la desigualdad socioeconómica, alcanzar la inclusión social, aumentar la participación ciudadana, fortalecer la democracia y reducir la asimetrías existentes, considerando la independencia de los Estados".

Además del Consejo de Jefes de Estado y del Consejo de Cancilleres y Delegados, la Unasur cuenta con doce Consejos Ministeriales Sectoriales, previstos en sus artículos $5^{\circ}$ y $6^{\circ}$ de su Tratado Constitutivo, como Consejos temáticos que respondan a los objetivos de este organismo. Dichos consejos son creados a través de la manifestación del Consejo de Jefes de Estado. Hace parte de los consejos existentes el Consejo de Defensa Sudamericano, creado en Salvador, en 2008.

La expresión “definiciones de políticas comunes” es la que particularmente nos llama más la atención. La construcción de ideas y expectativas suramericanas pasan por uno entendimiento compartido de los conceptos que, por su vez, permitan operacionalizar la cooperación (Saint-Pierre; Silva 2013). Este argumento es la base del esfuerzo del Unasur en primar por la univocidad conceptual de la Defensa en la región.

Desde la creación del Consejo de Defensa Suramericano, se consolida la dimensión institucional de la cooperación en defensa, a través de un proceso que va desde el nivel declaratorio de zonas de paz y de la implementación de medidas de transparencia y confianza mutua hasta la formación de un sistema de seguridad regional y cooperación en defensa, con la progresiva inclusión de definiciones, acuerdos y medios de cooperación propios de una institucionalidad regional que interactúe con las políticas y sistemas nacionales de defensa (Monteiro 2014, 392).

La creación del Centro de Estudios Estratégicos en Defensa (CEED), en 2010, significó un importante avance en este sentido. Este organismo objetiva "generar un pensamiento estratégico a nivel regional, que coadyuve a la coordinación y la armonización en materia de políticas de Defensa en Suramérica”, como está expuesto su carta (CEED 2011). El CEED es el único fórum permanente del Unasur y su constitución de hecho se da en mayo de 2011 en la República Argentina y en su Carta Constitutiva encontrase, entre otros objetivos, la construcción de una visión compartida que posibilite el abordaje común en materia de defensa y seguridad regional; la identificación de enfoques conceptuales y lineamientos básicos comunes que permitan la articulación de políticas en materia de defensa y seguridad regional y la concretización de medidas de Confianza Mutua.

En este marco, el CDS, por intermedio de los trabajos del CEED, publicó en 2012 en su llamado "Informe de Avance", un dibujo inicial de la definición regional de la Defesa: 
[...] la defensa constituye una función esencial del Estado, vinculada con la protección y la manutención de su soberanía e integridad de su población, territorios e instituciones; en tal virtud, abarca los asuntos de seguridad relacionados con el ámbito externo, en cuanto entornos estratégico de la política exterior e los Estados y se configura como el espacio específico y exclusivo de organización y uso de la fuerza militar del estado, en relación a riesgos y amenazas relativos a su integridad y existencia independiente y soberana ${ }^{5}(\operatorname{CDS} 2012)$.

Según Saint-Pierre y Silva (2013), el CEED realmente expresa el compromiso con la construcción compartida de Defensa para la ejecución de políticas regionales en este ámbito, pero difícilmente llegará a tanto. De la definición de Defensa regional del CEED se puede sacar algunas conclusiones preliminares. La primera es que el Consejo reconoce que hay, factualmente, diferencias conceptuales entre los países que necesitan ser dirimidas una vez que representan un obstáculo a la conducción de los trabajos del Consejo; y que el Centro, por su vez, es un semillero de producción académica, ideas y soluciones normativas para los problemas de la región. Tratase, por lo tanto, de un escenario a donde se puede crear valores conjuntos y alternativas críticas a las propuestas de Defensa de actores extra regionales. Un análisis detallado del informe trae, de cierta manera, las respuestas iniciales para la construcción de una identidad estratégica en los moldes planeados arriba por Luis Tibiletti (2014).

Según Rut Diamint (2014, 372), "la decisión de Brasil de crear un Consejo de Defensa en el marco del Unasur [...] ha obligado a fijar planes doctrinarios y de recursos de largo plazo en el campo de la defensa”. Es decir, la Defensa pasa a ser una construcción conjunta temática, a partir del proceso de institucionalización de lo cual se encargará el CDS, firmando un compromiso duradero entre las partes. Sin embargo, trabajar la Defensa regionalmente tiene sus impactos. Para Lima y Soares, la idea base de este compromiso seria "crear condiciones para una actuación más autónoma de la región en la cual está presente la potencia hegemónica mundial”.

Sobre la ausencia de los Estados Unidos en la región, Hal P. Klepak Powell (2013) escribe que este país no parece notar la región y que simplemente la América del Sur ya no se hace su prioridad. México, Centroamérica y Caribe en la actual coyuntura captan más atención de la superpotencia con las cuestiones de las drogas, del crimen organizado y de la migración ilegal que toda la América del Sur y que, de una manera o de otra, los ojos de Washington se voltearon para otras partes del mundo por cuestiones más o menos tradicionales. En cambio, para Jorge Battaglino (2008), las instituciones se vuelven un espacio clave para posicionarse, especialmente 
en un momento en que Estados Unidos y las principales potencias occidentales están ejerciendo una dominación casi monolítica. La profusión de ratificaciones y de iniciativas regionales de cooperación puede entenderse como una respuesta regional a la situación de unipolaridad y primacía que emerge en este sistema - la Unasur sería el más largo ejemplo en este sentido. Esto tiene, para el autor, contribuido considerablemente a la cooperación regional en defensa y seguridad.

Para Pablo Celi (2014), la Unasur tiene un papel fundamental en el rediseño de seguridad regional la América del Sur, incorporando una visión de inserción internacional, al avanzar en un proceso que articula la cooperación de los sectores de defensa de la región. Es importante, antes de señalarnos nuestros cuestionamientos en este proceso, lo que se caracteriza el término "región". Buzan y Waever explican este entendimiento por lo que nombran como "complejo regional de seguridad" que "se refiere al nivel en lo cual el Estado u otras instituciones son conectados de manera suficientemente cercana que sus seguridades no pueden ser consideradas separadas unas de las otras".

A fin de crear una pantalla de la región y dos desafíos del Unasur, los trabajos de João Fábio Bertonha (2013), Jorge Bataglino (2012) y Menezes Teixeira (2010) coinciden de manera sintética al dividir la región de la América del Sur en tres distintos escenarios estratégicos, cada uno marcado por sus particularidades. El primero sería el Cono Sur, compuesto principalmente por Argentina (siempre el ejemplo clave), Uruguay y Chile en que las fuerzas armadas de los dos primeros habrían perdido su capacidad real de disuasión y confiarían en mecanismos de respuesta mutua - como el Unasur - para cumplir su defensa; Chile, por su vez, al no descartar la posibilidad de conflicto con sus vecinos tiene cierta capacidad de actuación. De una manera general el Cono Sur es marcado por países relativamente estables, económicamente desarrollados, con un relativo bajo nivel de violencia interna y un control civil sobre los militares garantizando prácticamente no acceso a la vida y la seguridad públicas. La Zona Andina sería otra subregión con rasgos propios. Marcada por la instabilidad económica y siendo uno de los más grandes centros de producción de drogas del mundo, en ella se encuentran casos de acciones de guerrilla reales (Colombia) y potenciales (Perú), o sea, en que las Fuerzas Armadas actúan en la seguridad pública, más allá de pensar en temas como la soberanía o la preservación de intereses. Además de asimetrías de desarrollo económico y social, problemáticas como la indígena y poco grado de cooperación en términos de defensa, es la única zona que presenta tensiones factuales entre los países, aunque la posibilidad de conflicto real sea escasa: Colombia, Venezuela, Ecuador, Bolivia, Perú y Chile. Bertonha (2012) destaca Brasil como un 
caso aparte, que posee aspiraciones hegemónicas especiales, programas de desarrollo e inversiones en actualización y construcción de capacidades disuasivas. Adelante del rompecabezas suramericano, el autor que "no hay, ni podría haber de esta forma, una estrategia de defensa común en los países de la región. Cada Estado establece la suya de acuerdo a sus problemas internos a los recursos disponibles, o a su grado de dependencia del polo hegemónico americano y sus perspectivas y proyectos de inserción internacional. En nuestra visión, Brasil se constituye un caso aparte por la manera como se desvirtúa las misiones y empleo de sus Fuerzas Armadas lo que en oposición al que afirma Bertonha, pesa más en contra que a favor de la construcción de sus capacidades disuasorias.

En otra perspectiva, la coyuntura Buzan y Waever (2003), según lo cual el sub-complejo del Cono Sur - conformado por Argentina, Brasil, Paraguay, Uruguay, Chile y Bolivia, dirigido por la iniciativa regional de creación de una 'Organización Regional' que culminaría en la cohesión institucional y aquella, por principio, fundamentaría una comunidad de seguridad también merece ser ponderada, en el sentido de que es limitada la posibilidad de construcción de una comunidad de seguridad entre Estados que muestran distintas comprensiones sobre el uso de la fuerza militar en sus bases normativas y en sus acciones. En este sentido después de problematizarnos teóricamente la cuestión el uso de la fuerza, nos detendremos en una breve análisis comparada de los casos brasileño y argentino, puntuando las diferencias conceptuales en relación a la concepción entre Defensa y Seguridad y, en consecuencia de eso, al empleo de la fuerza militar que aún no separan del ideal de una visión estratégica suramericana. La continuación hasta los días actúales, de la instrumentalización de las Fuerzas Armadas brasileñas como fuerzas policiales, robustece un padrón exportado por los Estados Unidos para la América Latina en comienzo de los 1990, objetivando la desinstalación de capacidades militares tradicionales bajo el signo de su adaptación a los nuevos parámetros hemisféricos de seguridad, diseñado por las llamadas nuevas amenazas. Aún que inúmeras críticas ya fueran hechas a esto respecto, Brasil sigue reiterando esto patrón que, de cierto modo, mantén viva una cultura estratégica semejada por actor hegemónico extra regional, lo que justifica también la importancia de acompañar transformaciones en padrones de atribución de misiones a las fuerzas armadas regionales. Hablamos de una situación que se distingue de lo caso argentino, lo cual es caracterizado pela debida distinción entre la actuación en la seguridad pública e la defensa nacional, además de posicionar más asertivamente en favor de convergencias suramericanas acerca del uso de las fuerzas armadas, como se esboza abajo. 


\section{DEL MONOPOLIO DE LA FUERZA A LAS DISTINTAS COMPRENSIONES ESTATALES DE SU USO: LOS CASOS DE BRASIL Y ARGENTINA}

Stephan Walt, en 1991, definió Seguridad Internacional asegurando que ella " $[\ldots]$ explora las condiciones en las cuales el uso de la fuerza es más probable, las maneras en que esto de la fuerza afecta a los individuos, Estados y sociedades, así como las políticas específicas que los Estados adoptan para prepararse, prevenirse o declararen guerra" $(1991,67)$.

Saint-Pierre (2011) defiende que la naturaleza de la fuerza proyectada internamente es protectora de lo súbdito y se emplea en régimen de monopolio, lo que se puede conocer por "seguridad pública", "seguridad interna", "seguridad ciudadana”, etc. Y que "con efecto, el principal desafío y preocupación permanente del soberano es con el cuidado, por un lado, con la seguridad de sus súbditos y la manutención del orden que garantice el status quo frente a eventuales amenazas del conjunto de unidades decisorias" (Saint-Pierre 2011).

En sus propias palabras,

[...] la primera característica de este monopolio es que la naturaleza de la decisión y de la violencia para con los súbditos es ordenadora (en la medida en que se manifiesta normativamente) y protectora (tanto de los súbditos cuando del status quo normativo que les garantiza su seguridad). La naturaleza de la modalidad del empleo de monopolio legítimo de la fuerza para con los súbditos es de garantizar la seguridad y el orden, esto es, de anulación de la polémica interna y disolución del concepto de "enemigo" en el ámbito interno, una vez que su soberano debe a sus súbditos su protección y seguridad. De ahí que el ejercicio interno de la soberanía consista, ante cualquier cosa, en neutralizar los conflictos. Para su interior, el soberano es policía y, en el sentido estricto del término, solamente para el exterior es política (Saint-Pierre 2011, 7). ${ }^{6}$

Es sobre esta misma diferenciación, inclusive, en que surgen las definiciones conceptuales de Seguridad Interna e Defesa, presentes en la mayoría de las Constituciones Nacionales bajo la luz de la percepción de lo que cada Estado entiende sobre los respectivos temas. ${ }^{7}$

Por otro lado, el interés nacional es posiblemente el aspecto más importante para que posamos definir la lectura que el estado hace de su papel delante el mundo y del entorno con lo cual se relaciona. El interés modula los objetivos de actuación externa de largo plazo aunque a veces la conjuntura internacional influya en la agenda gubernamental (Battaglino 2008; Vera 2009). Es con base en esta lectura de objetivos e intereses que los estados crean sus aparatos institucionales - ministerios, documentos declarativos, 
rol de las fuerzas armadas, etc. En el caso de las políticas de defensa y todo lo que vimos que ellas representan, pueden variar, de país para país, los agentes predominantes en su elaboración: en general militares, agentes ejecutivos y parlamentarios (Winand; Saint-Pierre 2007; Pion; Ugarte 2013).

El grado de intervención que cada uno de estos grupos pueden ejercer es otro factor determinante de las percepciones contenidas en las leyes y documentos nacionales, lo que hace con que los entendimientos sobre la defensa varíen en una misma región.

Elucidamos este razonamiento basado en breve comparación entre dos casos: el caso argentino, es reconocido por el contenido más político de los mecanismos institucionales que lo componen, así como por una más precisa diferenciación entre seguridad interna y defensa, que es visible en las atribuciones del Ministerio de la Seguridad Interior y el de la Defensa. Ya el brasileño, es marcado pela recurrente intervención - o convocación de intervención - de los militares en la seguridad pública. Las fuerzas armadas brasileras siguen presentes en la vida pública interna (Winand; Saint-Pierre 2007). Estos dos casos nos dan el gancho para la discusión sobre la dilución entre la seguridad y la defensa en los países de la América del Sur y lo que puede ser entendido como la ampliación del campo de actuación de las fuerzas armadas de la región. Pues, al fin y al cabo, como las fuerzas armadas son los agentes ejecutores de la defensa, pero que por su propia característica organizacional y que por gozaren todavía de autonomía bastante para conducir aspectos de la política de defensa a partir de su propia visión del mundo, observar su actuación significa observar rumbo a donde la región está yendo en términos de construcción de una aspiración compartida.

Abajo nos enfocaremos en como Brasil y Argentina enuncian sus objetivos de Defensa en sus marcos normativos, llevando en cuenta que los mismos, como afirmado por Soares (2013), permiten la compresión de las percepciones sobre prioridades estratégicas, y la observación de como los países auto refieren en sus políticas, además de contribuyeren para analizar el comportamiento de los actores, y así ponderar en qué medida su cultura estratégica se vuelta a una referencia colectiva.

Con respecto a Brasil, la actual Política de Defensa Nacional, del año de 2012, entiende que la Defensa, en sí mismo, es:

El conjunto de medidas y acciones del Estado, con énfasis en el campo militar, para la defensa del territorio, la soberanía y los intereses nacionales contra amenazas externas preponderantemente potenciales o manifiestos (Brasil 2012).

Ha sido señalado que el uso del término "preponderantemente" al contrario de otros, a ejemplo de "exclusivamente" abre márgenes para el uso 
equivocado de los medios de la defensa a fines cuya esencia no es de política exterior, a ejemplo de Soares (2008), Winand y Saint-Pierre (2010). Corroborando con nuestro raciocinio, por otro lado, los militares siguen interiorizando una identidad de fuerza de seguridad pública, al contrario de las fuerzas argentinas, como mostramos a seguir. ${ }^{8}$

En la Estrategia de Defensa Nacional (END) se establecen directrices para la preparación y formación de las Fuerzas Armadas asegurando el funcionamiento de la defensa y el cumplimiento de sus metas, pero no delinea claramente lo que concibe para la "seguridad nacional", lo que también permite la extrapolación de las funciones primarias de la Defensa.

En distinta dirección, en Argentina, además de la Ley de Defensa Nacional de 1988, la Directiva Política de Defensa Nacional, un documento oficial del Gobierno Kirchner todavía vigente, otorgado por el Decreto 1714 de 2009, establece que Argentina sea conducida por la "defensiva estratégica", lo que cede lugar a la derogación de "hipótesis de conflicto" con los países vecinos, como garantiza el documento, así como neutraliza la presunción de “posiciones de agresión o expansión”. Además, el concepto de la defensa nacional definido en la Ley n. 23.554, de 26 de abril de 1988, en concreto, en el apartado 2 del artículo 1, sostiene que:

[...] la integración y la acción coordinada de todas las fuerzas de la nación para resolver esos conflictos que requieren el uso de las Fuerzas Armadas, en forma disuasiva o efectiva para hacer frente a los ataques procedentes de fuentes externas (Argentina 1988).

Dos diferencias notables que ya se pueden identificar por la forma en cómo es la conceptualización dada por los dos países: una dimensión en la que se entiende Defensa, y el otro por el objeto, que se dedica a la defensa. Mientras que Brasil corta como objetos de su soberanía política de defensa, el territorio y sus intereses nacionales, prestando el concepto patrimonialista un tono, la Política de Defensa de Argentina se dedica a resolver las agresiones externas. Y mientras que Argentina lo hace único al ambiente externo, Brasil abre la prerrogativa seria para el uso interno de las fuerzas armadas. Sintetizamos la comparación de los objetivos de Defensa de ambos países en la siguiente tabla: 
\begin{tabular}{|l|}
\hline Brasil: Decreto n. 5484, de 2005. \\
Política de Defensa Nacional (PDN) (Art. 5)
\end{tabular}

I. Asegurar la soberanía, patrimonio nacional y la integridad territorial;

II. Defender los intereses nacionales y las personas, los bienes y los recursos brasileños en el exterior;

III. Contribuir a la preservación de la cohesión y la unidad nacional;

IV. Contribuir a la estabilidad regional;

V. Contribuyen al mantenimiento de la paz y la seguridad internacionales;

VI. Intensificar la proyección de Brasil en el concierto de las naciones y su mayor inclusión en la toma de decisiones a nivel internacional;

VII. Mantener las fuerzas armadas modernas, integrado, capacitado y equilibrada, con el aumento de la profesionalización, que operan de forma conjunta y debidamente desplegados en todo el territorio nacional;

VIII. Educar a la sociedad brasileña de la importancia de los asuntos de defensa nacional;

IX. Desarrollar la industria de defensa nacional, centrado en el logro de la autonomía en tecnologías indispensables;

X. Estructuración de las Fuerzas Armadas en los alrededores de capacidades, dotándolos de personal y material en consonancia con la planificación estratégica y operativa; y

XI. El desarrollo del potencial de la logística de defensa y movilización nacional.
Argentina: Ley n. 23.554, en 1988.

Ley de Defensa Nacional (Art. 8)

1. Determinar las posibilidades de conflicto y debería mantenerse como una hipótesis de guerra;

2. Desarrollar hipótesis de guerra, que se establece para cada uno de ellos los medios que se utilizarán;

3. Desarrollar planes que permiten una adecuada preparación de toda la nación para una posible guerra;

4. Desarrollar planes para la realización de los niveles de defensa nacional, que corresponde a la estrategia militar y la estrategia operativa;

5. Conducir la guerra en todos sus aspectos, desde el nivel de la estrategia nacional;

6. Conducción de las Fuerzas Armadas y los sectores de esfuerzos afectadas por el conflicto, el nivel estratégico y operativo militar estratégica del país;

7. Desarrollar y poner en práctica la movilización nacional;

8. Asegurar la ejecución de las operaciones militares conjuntas de las fuerzas armadas y las operaciones combinadas que pudiera surgir;

9. Coloque la hipótesis de convergencia para permitir la preparación de alianzas suficientes necesarios para responder de manera apropiada a la posible realización de la hipótesis de la guerra;

10. Verificar las acciones de guerra.

Figura 1 - Tabla comparativa de los objetivos de Defensa Nacional de Brasil y Argentina. ${ }^{9}$

Es posible observar que los objetivos brasileños tienen un carácter mucho más general, mientras que los argentinos se centran claramente en la detección y contención de las amenazas a través de un plan específico para ellos. Por otra parte, mientras que Brasil expone la proyección de la pretensión de la región y el mundo, Argentina asume la necesidad de buscar alianzas estratégicas específicas y permanentes para cumplir con su defen- 
sa. En las palabras de Lima y Soares, "mientras que Argentina otorga peso relevante a la defensa regional, Brasil se esfuerza en lograr una inserción en el nivel global y el peso de Defensa cae sobre sus propios recursos." 10

Por su vez, Jorge Battaglino (2008) resume que hubo una ampliación del rol de las Fuerzas Armadas (a parte de la tradicional - o epistemológica - que trabajamos arriba) por especialmente tres factores: el predominio de conflictos internos en algunos países - como Colombia y Perú; la relativización del principio de la soberanía - de procedencia internacional, reconociendo la insurgencia de debates como seguridad humana, amenazas multidimensionales e intervención internacional en el pos Guerra Fría, más allá de la Defensa; y la primacía militar y la doctrina del ataque preventivo - respuesta de los Estados Unidos en el contexto de estos debates.

En que concierne específicamente el uso interno de las fuerzas armadas, Saint-Pirre y Donadelli (2016) observan que "lo miedo y la inseguridad tomaran cuenta de las sociedades latino-americanas" y que recursos humanos y financieros fueran desviados para auxiliar otras estructuras institucionales. Entretanto, D’Araújo (2013) llama la atención para las misiones de paz que son actividades qué, a pesar de consideradas subsidiarias, son importantes para sostenimiento del imagen del Estado y su proyección, en otros términos, están relacionadas a la política exterior. Datos provenientes de los Atlas de la Resdal (2012, 2014, 2016) demuestran que en cuestiones de misiones de paz, en 2014 la Argentina aportaba 13,72\% del total de efectivo latinoamericanos dispuestos en misiones de paz de la ONU a la fecha, en cuanto las fuerzas brasileñas representan el 27,7\%. Juntos, Brasil y Argentina, en 2016, están presentes en varias de las Misiones de Paz existentes, a saber Sahara Occidental, República Centroafricana, Chipre, Congo, Liberia, Costa del Marfin, Sudán del Sur, Abiel y Haití, siendo esta encabezada por el comando brasileño.

En la Figura 2, recuperamos las principales misiones subsidiarias de Brasil y Argentina, porque más allá de las principales han ganado recurrente importancia al tener empleo de sus respectivas Fuerzas Armadas para estos fines. De los Atlas también se encuentra bastantes datos sobre el empleo de las fuerzas armadas brasileñas en la seguridad de grandes eventos, como el Mundial FIFA, en IV Encuentro de los BRICs y las Olimpíadas de Río de Janeiro. En el caso argentino, en apoyo logísticos a varios ministerios, como de Salud y Desarrollo en campañas internas del país. Estas dos temáticas, apoyo a misiones de paz y operaciones internas en cooperación con otros ministerios representan temas compartidos entre los dos países. Por otro lado, los casos de "Garantía de Ley y Orden" (GLOs) en los conocidos eventos de "pacificación” de favelas en Brasil, demuestra el claro (RESDAL 2012, 2014 y 2016). Situación vetada por ley 
en el caso argentino. Eso lleva a Juan Rial (2013, 32) a argumentar que "los militares cumplen misiones en el interior de las fronteras, sea para apoyar el proceso de modernización o para cubrir vacíos en la capacidad de gestión del Estado en otros ámbitos, así como para mantener el orden público. La discusión pública del amplio margen de acción del aparato armado del Estado es sustancial” (Rial 2013, 32).

\begin{tabular}{|c|c|}
\hline República Argentina & República Federativa do Brasil \\
\hline $\begin{array}{l}\text { ización y } \\
\text { las Fuerzas } \\
\text { o de Defensa } \\
\text { de 2006) }\end{array}$ & 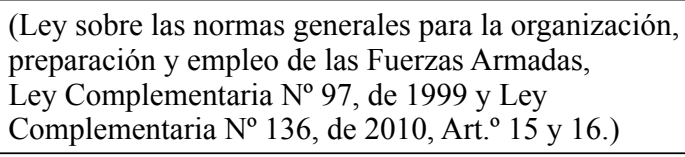 \\
\hline $\begin{array}{l}\text { Misiones subsidiarias: } \\
\text { - Participar de las operaciones } \\
\text { multinacionales en el marco } \\
\text { de las Naciones Unidas } \\
\text { - Participar en operaciones } \\
\text { de seguridad interior, según } \\
\text { lo establecido por la Ley N }{ }^{\circ} \\
24.059 \\
\text { - Apoyar la comunidad nacional } \\
\text { o de países amigos } \\
\text { - Participar de la construcción } \\
\text { de un sistema de defesa subre- } \\
\text { gional }\end{array}$ & $\begin{array}{l}\text { Misiones subsidiarias: } \\
\text { - Participación en operaciones de paz } \\
\text { - Cooperación con el desarrollo nacional y la defensa } \\
\text { civil } \\
\text { - Participación en campañas institucionales de utili- } \\
\text { dad pública y o de interés nacional } \\
\text { - Corresponde a las Fuerzas Armadas, entre otras } \\
\text { actividades pertinentes, así como sus atribucio- } \\
\text { nes complementares y preservadas las competen- } \\
\text { cias exclusivas de la policía judiciaria, actuar por } \\
\text { medio de medidas preventivas y represivas en las } \\
\text { regiones fronterizas del mar y aguas interiores, } \\
\text { independiente de pose, propiedad o destino, con- } \\
\text { tra delitos fronterizos y ambientales: I - patrullaje, } \\
\text { II - revisión de personas, vehículos terrestres, em- } \\
\text { barcaciones y aeronaves, III - detener en flagrante } \\
\text { delito }\end{array}$ \\
\hline
\end{tabular}

Figura 2 - Tabla comparativa de misiones de las FFAA de Brasil y Argentina. ${ }^{11}$

En que pese el punto llave de que las fuerzas armadas de Brasil y Argentina han cumplido misiones diferentes, principalmente cuándo se compara el enfoque en empleo interno, las misiones de paz constituyen un lugar común en lo cual ambos los países pueden definir intereses, objetivos y acciones compartidas y a partir de ellas reinventar nuevos conceptos y concepciones, tanto desde el punto de vista político como de los actores militares que experimentan directamente de estas prácticas. 


\section{CONSIDERACIONES FINALES}

Pudimos observar que, se por un lado, el CDS tiene una misión noble en la conformación de una identidad, de una política y de una real cooperación en defensa - como plasman sus objetivos - por otro, las distintas fuerzas armadas de los países que componen la región se ubican en diferentes realidades, actuando en distintas funciones y buscando objetivos tan dispares que cualquier tentativa de amalgamación por parte de CDS va a sonar superficial. Al reflexionar sobre esta temática regionalmente no estamos ejercitando solamente las estructuras teóricas que disponen las Relaciones Internacionales. Estamos discutiendo nuestro propio espacio, nuestros modelos de inserción y la propia significación que tiene el camino que hemos elegido conjuntamente. Como advierte Comini (2013, 17): "los asuntos de defensa, por su vinculación con la supervivencia e independencia de los Estados, siempre se han cruzado con un recelo particular cuando de procesos de integración se ha tratado". Sin embargo, el esfuerzo unasurino de intentar conjugar los diferentes países de la región rumbo a un futuro común es el primero paso en esta caminada. Fue prácticamente unánime entre os distintos autores que usamos en este trabajo que la Unasur vino para ocupar un lugar no solo en la política externa de los países que componen la región, sino el propio papel que la región tiene el mundo.

Para Nicolás Comini (2015), "puede concluirse que, aún sobre la base de múltiples disensos y de perspectivas encontradas que llevaban hacia la divergencia, [...] (el Consejo) basaba su razón de ser en la identificación de mínimos comunes denominadores; es decir aquellos puntos que motivaban la convergencia”. No obstante, el hecho de que el CDS traiga un nuevo nivel a los debates de la Defensa no quiere decir que eso haya sanado los viejos desentendimientos sobre el tema. Por lo tanto, los esfuerzos del CDS se chocan no solo con las pretensiones e intereses individuales de cada país de la región, pero con asimetrías, diferentes entendimientos y muchas veces la indiferenciación de los roles de seguridad y defensa en la región, los cuales confunden no solo los roles de las fuerzas armadas y la posibilidad de actuación común como también tornan esta actuación incoherente en varios aspectos: en contenido, en método y en motivación.

Las fuerzas armadas y sus misiones como elementos clave en la comprensión del tema (Marques 2007, Domingos Neto 2013), sí, relevan los elementos que constituyen el espectro estratégico de cada país (Soares 2013) y la complejidad que tenemos en cuanto región. Al buscar alinear los entendimientos, aunque a través de mínimos indicadores comunes, el CDS contribuye sin precedentes cuando permite que cada uno de los "yo" regionales tenga la palabra en la conformación de un "nosotros" (Soares 
2013, Soares; Lima 2013). A despecho de las divergencias apuntadas, los intereses de Brasil y de Argentina, bien como sus similares aspiraciones reveladas por los documentos de Defensa, en respecto a las misiones de paz muestran que hay "denominadores comunes" que deben der explorados.

Si aún falta mucho trabajo - y para eso está el CEED/CDS/ UNASUR - para que entre nosotros alcancemos la univocidad conceptual (Saint-Pierre; Lopes 2013) en todos los temas que cómo región nos dicen respeto en sí ya estamos caminando hacia la conformación de una región por el simple ejercicio de pensar a sí mismo. A final, si cómo plantea Luis Tibiletti (2014), los puntos en donde las modificaciones en las concepciones prácticas pueden modificar identidades estratégicas son los más importantes en ser investigados, es muy acertado que el CDS los tenga en claro para llevar a cabo su objetivo de una arquitectura regional.

\section{REFERENCIAS}

Battaglino, J. 2010. Rearme y baja percepción de amenaza interestatal en Sudamérica. ¿Es posible tal coexistencia? Perfiles Latinoamericanos, 35, 61-87.

Battaglino, J. 2012. Defence in a Post-hegemonic regional agenda: The case of the South American Defence Council. In: Riggirozzi, P., Tussie, D. The Rise of Post-hegemonic Regionalism. New York: Springer.

Berlin, D. P., Ugarte, J. M. 2013. Introducción. In: Berlin, D. P., Ugarte, J. M. Organización de la defensa y control civil de las Fuerzas Armadas en América Latina. Buenos Aires: Jorge Baudino.

Bertonha, J. F. 2012. ¿Seguridad o defensa? Dilemas de las Fuerzas Armadas latinoamericanas en los comienzos del siglo XXI? Relaciones Internacionales, 43, 19-37.

Buzan, B., Hansen, L. 2009. The Evolution of International Security Studies. Cambridge: Cambridge University Press.

CEED. 2010. Estatuto del Centro de Estudios Estrategicos de Defensa del Consejo de Defensa Sudamericano. Disponível em: http://www.ceedcds.org.ar/ Espanol/o1-CEED/04-Estatuto-Ceed.html. Acessado em: 9 fev. 2016.

Celi, P. 2014. Defesa e segurança regional na América do Sul. In: Monteiro, Á. D., Winand, É. C. A., Goldoni, L. R. F. Defesa da Amazônia: VII ENABED. São Cristóvão: Editora UFS.

Comini, N. 2013. ¿De quién se defiende Sudamérica? Foreign Affairs Latinoamérica, 13 (1), 16-24. 
Comini, N. 2015. El origen del Consejo de Defensa Suramericano. Modelos en pugna desde una perspectiva argentina. Revista de Estudios en Seguridad Internacional, 1 (2) 109-135.

Comini, N. 2016. SuRamericanizados. La integración regional desde la Alianza al Kirchenerismo. Buenos Aires: Ediciones Universidad del Salvador.

Diamint, R. 2013. La política de defensa en Argentina: lecciones nacionales y regionales. In: Carmo, C. A. do., Winand, É. C. A., Barnabé, I. R., Pinheiro, L. M. (Ed.). Relações Internacionais: olhares cruzados. Brasília: FUNAG.

Donadio, M. 2010. El Consejo Sudamericano de Defensa y papel en el desarrollo de la confianza mútua. In: CEED. Confianza y Seguridad en America del Sur. Quito: Publicación del Consejo de Defensa Suramericano.

Marques, A. A. 2007. Amazônia: pensamento e presença militar. Tesis doctoral. São Paulo, Universidade de São Paulo.

Martínez, R., D’Araujo, M. C., Rial, J., Salomón, L. 2013. Las relaciones civiles-militares en sociedades en transformación: América Latina. In: Documentos CIDOB América Latina, 36.

Meneses, A. W. T. 2010. Regionalismo y seguridad sudamericana: ¿son relevantes el Mercosur y la Unasur? Íconos. Revista de Ciencias Sociales, 14 (3), 41-53.

Meza, M. N. 2009. Política Exterior y Política de Defensa. Una cercanía esquiva. Revista Política y Estrategia, Santiago, 114, 54-99.

Montoya, A. C. P., Saint-Pierre, H. L. 2014. As medidas de confiança no Conselho de Defesa Sul-americano (CDS): análise dos gastos em defesa (2009-2012). Revista Brasileira de Política Internacional, 57 (1), 22-39.

Neto, M. D. 2013. A necessidade dos Estudos de Defesa. In: Monteiro, Á. D., Winand, É. C. A., Goldoni, L. R. F. (Ed.). Pensamento Brasileiro em Defesa: VI ENABED. Aracaju: Editora UFS.

Ponte, A. da. 2013. De la Política tecnológica de defensa a los desafíos de la cooperación regional. Revista da Escola de Guerra Naval, Rio de Janeiro, 19 (1).

Powel, H. P. K. 2013. El contexto actual de la defensa suramericana: una percepción desde afuera. In: Monteiro, Á. D., Winand, É. C. A., Goldoni, L. R. F. (Ed.). Pensamento Brasileiro em Defesa: VI ENABED, 247-258. Aracaju: Editora UFS.

RESDAL. 2012. Atlas Comparativo de Defensa y Seguridad en América Latina. Buenos Aires: RESDAL. 
RESDAL. 2014. Atlas Comparativo de Defensa y Seguridad en América Latina. Buenos Aires: RESDAL.

RESDAL. 2016. Atlas Comparativo de Defensa y Seguridad en América Latina. Buenos Aires: RESDAL.

Saint-Pierre, H. L. 2011. "Defesa" ou "Segurança"? Reflexões em torno de Conceitos e Ideologias. Contexto Internacional, 33, 407-435, jul./dic.

Saint-Pierre, H. L. 2013. Reflexiones sobre los estudios de defensa: perspectiva filosófica e histórica. In: Mora, R., Jesús, A. (Ed.). Ciencias que uniforman el medio militar. Bogotá: Sello Editorial ESMIC.

Saint-Pierre, H. L., Donadelli, L. 2016. As atribuições das Forças Armadas nos países sul-americanos. In: Winand, É. C. A., Rodrigues, T., Aguilar, S. (Ed.). Defesa e segurança do Atlântico Sul: VIII ENABED. São Cristóvão: Editora UFS.

Saint-Pierre, H. L., Silva, D. L. 2013. A Torre de Babel sul-americana: a importância da convergência conceitual para a cooperação em Defesa. In: Carmo, C. A. do, Winand, É. C. A., Barnabé, I. R., Pinheiro, L. M. (Ed.). Relações Internacionais: olhares cruzados, Brasília: FUNAG.

Saint-Pierre, H. L., Silva, D. L. 2014. Percepções de segurança regional no âmbito da UNASUL: O Conselho de Defesa Sul-Americano. In: Ayerbe, L. (Ed.). Territorialidades e entrecruzamentos geopolíticos na América Latina. São Paulo: Editora Cultura Acadêmica.

Silvestre, C. A. D. G. 2015. Brasil e Argentina no âmbito do Conselho de Defesa Sul-Americano: por uma convergência de conceitos e agendas. Tesis de grado. São Cristóvão, Universidade Federal de Sergipe (UFS).

Soares, S. A. 2013. Identidade entre Argentina e Brasil: compassos e descompassos na cooperação em Defesa. In: Anais dos $4^{\circ}$ Encontro Nacional da Associação Brasileira de Relações Internacionais, 1-20.

Soares, S. A., Lima, R. C. 2013. No limbo da dissonância: Argentina e Brasil no campo da Defesa. In: Carmo, C. A. do., Winand, É. C. A., Barnabé, I. R., Pinheiro, L. M. (Ed.). Relações Internacionais: olhares cruzados. Brasília: FUNAG.

Soares, S. A., Prado, M. do., Godoy, D. B. P. de. 2014. Rumo ao Eldorado: uma viagem de 20 anos no campo da Defesa na América do Sul. In: Monteiro, Á. D., Winand, É. C. A., Goldoni, L. R. F. (Ed.). Defesa da Amazônia: VII ENABED. São Cristóvão: Editora UFS. 
Tibiletti, L. 2014. Las distintas ofertas de identidades estratégicas en los países de UNASUR y su impacto en la búsqueda de una identidad de defensa suramericana. Revista Brasileira de Estudos de Defesa (RBED), 1, 13-37, jul./dez.

Vera, J. F. 2009. La ciencia política y los estudios sobre seguridad y defensa. Revista Política y Estrategia, Santiago, 114, 43-54.

Wendt, A. 1994. Collective Identity Formation And The International State. The American Political Science Review, 88 (2), 384-396.

Winand, É. C. A. 2017. Diplomacia e Defesa na gestão Fernando Henrique Cardoso (1995-2002): história e conjuntura na análise das relações com a Argentina. São Paulo: Editora UNESP.

Winand, É. C. A., Saint-Pierre, H. L. 2007. O legado da transição na agenda democrática para a defesa: os casos brasileiro e argentino. In: Saint-Pierre, H. L. Controle civil sobre os militares e política de defesa na Argentina, Brasil, no Chile e no Uruguai. São Paulo: Editora UNESP: Programa San Tiago Dantas de Pós-Graduação em Relações Internacionais da UNESP, Unicamp e PUC-SP. 


\section{NOTAS}

1. "Um conceito categoriza, define, limita, a realidade em torno de uma hipótese formulada por um ser histórico e social".

2. En lo que hace referencia específicamente a Brasil y Argentina, Soares y Lima (2013) defienden que hay entre los dos países "un limbo disonante", conformado por la combinación entre culturas estratégicas "reciprocas". Esto solamente será superado, según los autores, cuando la reciprocidad sea suprimida por una "cultura estratégica conjunta", una vez que en la relación de reciprocidad, es decir, cuando ambos los países elaboran sus visiones teniendo el "otro" como referente, la relación directa que establecen entre sí puede ser de rivalidad, competencia o de amistad. Cuanto, sin embargo, la representaciones dejan de se orientar por la visión recíproca, o mejor, cuando el "otro" deja de ser el referente, acciones coordenadas pasan a ser favorecidas, sobre todo porque la desconfianza aún está presente en la dinámica de reciprocidad entre los dos.

3. Saint-Pierre (2013) define claramente que hay dos maneras de se observar la defensa: pragmáticamente y epistemológicamente. No hay necesariamente una forma correcta y una equivocada: hay diferentes objetivos para cada una de estas perspectivas.

4. "Em suma, a cultura estratégica de um grupo social é moldada pela prática de seus membros bem como a prática dos membros de um grupo social é moldada pela cultura estratégica desse grupo".

5. "A defesa constitui uma função essencial do Estado, vinculada com a proteção e a manutenção de sua soberania e integridade de sua população, territórios e instituições; em tal virtude, abarca os assuntos de segurança relacionados com o âmbito externo, enquanto entorno estratégico da política Exterior dos Estados e se configura como o espaço específico e exclusivo de organizações e uso da força militar do Estado, em relação a riscos ou ameaças relativos à sua integridade e existência independente e soberana."

6. “[...] uma primeira característica desse monopólio é que a natureza da decisão e da violência para com os súditos é ordenadora (na medida em que se manifesta normativamente) e protetora (tanto dos súditos quanto do status quo normativo que garante a segurança deles). A natureza da modalidade do emprego do monopólio legítimo da força para com os súditos é de garantir a segurança e a ordem, isto é, de anulação da polêmica interna e dissolução do conceito do "inimigo" no âmbito interno, uma vez que seu soberano deve aos seus súditos sua proteção e segurança. Daí que o exercício interno da soberania consista, antes de mais nada, em neutralizar os conflitos [...]. Para o seu interior, o 
soberano é polícia, e no sentido estrito do termo, apenas para o exterior é política" $(2013,7)$.

7. Vea también Saint-Pierre en Atlas Comparativo de Defensa y Seguridad en América Latina (Resdal 2012, 44).

8. Es interesante retomar la discusión sobre la identidad de las fuerzas armadas brasileñas en la última década del siglo XX. Como puntúan Winand y Saint-Pierre (2007), no obstante el fin de la guerra fría prenunciase nuevas prioridades, se quedaba imposible que los militares revisasen sus valores de la noche por el día, así como sus conceptos estratégicos históricamente construidos. Después de muchas décadas de combate al enemigo interno, se tornaran desfasadas la doctrina y la logística.

El enemigo interno so fuera sustituido por una misión de igual relevancia para el Estado, al comienzo de los años 2000, con la participación de las fuerzas de paz junto a la MINUSTAH, lo que costuró el sentido de noble misión a un gran propósito de política exterior. Con todo, lo que se supo cómo una revelación del talento de las fuerzas brasileñas de paz fue utilizado por parte de los atores como argumento para la continuidad de las acciones policiales junto a los morros nos cuales se sedeaban las células do tráfico de drogas: según ellos, el know how adquirido en los morros habilitaban los militares a conformaren las fuerzas de paz que irían al Haiti. En sentido de retroalimentación, la experiencia adquirida en Haiti hacía, supostamente, de los militares brasilenõs, los agentes más preparados para contener el tráfico.

9. Silvestre $(2012,16)$. Elaborada con base en los documentos de referencia. 10. "[ ...] enquanto que a Argentina outorga peso relevante à defesa regional, Brasil se esforça em conseguir uma inserção em nível global e o peso da Defesa recai sobre seus próprios recursos (Lima; Soares 2014, 346).

11. Silvestre $(2012,29)$. Elaborada con base en los documentos de referencia. 


\section{ENTRE PUENTES Y MUROS. EL CONSEJO DE DEFENSA SUDAMERICANO Y LAS IDENTIDADES ESTRATÉGICAS DE BRASIL Y ARGENTINA.}

\section{RESUMEN}

Las relaciones que los países de la región pueden ejercer pasan esencialmente por la definición de conceptos y concepciones o, en otras palabras, sus miradas. En este trabajo intentamos mostrar que la divergencia entre los modos de pensar la Defensa entre los países y la amplitud de las nuevas misiones que han asumido las fuerzas armadas de la región complican la posibilidad de un escenario regional convergente como plantea el Consejo de Defensa Sudamericano. Como manera de ponerle luz a dichas diferencias, realizaremos una breve comparación entre los casos de Brasil y Argentina.

Palabras-clave: América del Sur; Unasur; CDS; Defensa.

\section{ABSTRACT}

The regional cooperation on Defense sector is conditioned by the achievement of a common understanding about strategic interests and challenges. This paper intends to show how conceptual divergences followed by an imprecise and wide scope of the new missions of the South American Armed Forces can constitute a serious obstacle to the development of the South American Defense Council. We will briefly compare the cases of Brazil and Argentina in order to show the differences that come out between then.

Keywords: South America; Unasur; CDS; Defense. 